\title{
Análisis geográfico de la mortalidad de diferentes usuarios de vías de tránsito y factores de área asociados en Argentina
}

\section{Geographic analysis of the mortality of different road users and associated area factors in Argentina}

\author{
Carlos Marcelo Leveau ${ }^{1,2}$ (D) \\ ${ }^{1}$ Instituto de Salud Colectiva, Universidad Nacional de Lanús - Remedios de Escalada, Lanús, Argentina. \\ ${ }^{2}$ Consejo Nacional de Investigaciones Científicas y Técnicas (CONICET), Buenos Aires, Argentina.
}

Cómo cita: Leveau CM. Análisis geográfico de la mortalidad de diferentes usuarios de vías de tránsito y factores de área asociados en Argentina. Cad Saúde Colet, 2020;28(2):165-179. https://doi.org/10.1590/1414-462X201900020057

\begin{abstract}
Resumen
Introducción: Las muertes por lesiones de tránsito son la principal causa de muerte por lesiones en la población de 5-64 años en Argentina. Objetivo: Describir las variaciones espaciales del riesgo de mortalidad de diferentes tipos de usuarios de vías de tránsito y analizar factores de área asociados con el riesgo de mortalidad en Argentina durante los períodos 1999-2003 y 2008-2012. Método: Se realizó un estudio ecológico espacial utilizando los departamentos/partidos como unidades de análisis. Se consideraron las muertes de peatones, usuarios de bicicleta, motocicleta y automóvil y usuarios no especificados de vías de tránsito. Como variables independientes se seleccionaron variables sociodemográficas y variables de patentamiento automotor. Resultados: El riesgo de mortalidad de los usuarios de vías de tránsito se concentró espacialmente durante los dos períodos en Argentina. El nivel socioeconómico bajo a nivel departamental estuvo relacionado a un riesgo alto de mortalidad en peatones y usuarios de bicicleta. En áreas con mayor patentamiento de nuevos automóviles se observó un mayor riesgo de mortalidad en usuarios de bicicletas durante 1999-2003. Conclusión: Las grandes disparidades en la distribución geográfica del riesgo de mortalidad de diferentes usuarios de vías de tránsito indican la adopción de políticas regionales de seguridad vial diferenciales en Argentina.
\end{abstract}

Palabras-clave: análisis espacial; mortalidad; lesiones de tránsito; regresión espacial; Sistemas de Información Geográfica.

\begin{abstract}
Background: Traffic injury deaths are the leading cause of injury death in the 5-64-year-old population in Argentina. Objective: To describe the spatial variations of the risk of mortality of different types of road users and to analyze area factors associated with the risk of mortality in Argentina during the periods 1999-2003 and 2008-2012. Method: A spatial ecological study was carried out using the departments/parties as spatial units of analysis. The deaths of pedestrians, bicycle, motorcycle, and automobile users, and unspecified road users were considered. Sociodemographic characteristics of area and variables related to automotive patenting were the independent variables. Results: The mortality risk of road users was spatially concentrated during the two periods in Argentina. The low socioeconomic level at the departmental level was related to a high risk of mortality in pedestrians and bicycle users. In areas with higher patenting of new cars, a higher risk of mortality was observed in bicycle users during 1999-2003. Conclusion: The great disparities in the geographical distribution of the mortality risk of different road users indicated the adoption of different regional road safety policies at the national level.
\end{abstract}

Keywords: spatial analysis; mortality; traffic injuries; spatial regression; Geographic Information Systems.

Este es un artículo publicado en acceso abierto (Open Access) bajo la licencia Creative Commons Attribution, que permite su uso, distribución y reproducción en cualquier medio, sin restricciones siempre que el trabajo original sea debidamente citado.
Trabajo realizado en la República Argentina.

Correspondencia: Carlos Marcelo Leveau. E-mail: cmleveau@hotmail.com

Apoyo financiero: ninguno.

Conflicto de intereses: nada que declarar.

Recibido em: Mar. 14, 2018. Aprobado em: Ago. 04, 2019 


\section{INTRODUCCIÓN}

Las muertes por tránsito constituyen un problema mundial de salud pública a nivel global: aproximadamente más de 1,2 millones de personas mueren cada año como consecuencia de un accidente de tránsito, de los cuales un $91 \%$ corresponde a países de ingresos bajos y medios. En Argentina las muertes por accidentes de tránsito ocupan el primer lugar en el grupo etario de los 5 a los 64 años, entre todas las muertes debidas a causas externas'. Ante este panorama preocupante, se requieren estudios epidemiológicos enfocados en los diferentes usuarios de las vías de tránsito. El mapeo de la mortalidad en estos usuarios permite registrar la existencia de diferentes patrones de distribución espacial para cada usuario y formular nuevas hipótesis acerca de los factores causales de las distribuciones geográficas resultantes.

Desde el enfoque de Haddon ${ }^{2}$ se considera a los accidentes de tránsito como un fenómeno complejo en el que intervienen tres dimensiones: persona, vehículo y medio ambiente (físico y social). Desde Latinoamérica, la perspectiva de la determinación social en salud postula que los niveles biológico y psicológico se encuentran subsumidos en el nivel social ${ }^{3}$. De esta manera, las desigualdades sociales en la salud se reflejarían en desigualdades territoriales. Por lo tanto, el estudio del contexto en el que se produce una muerte por lesión de tránsito es de suma importancia para la comprensión de estos fenómenos. En los países desarrollados se ha encontrado que, controlando por la posición socioeconómica a nivel individual, existe un mayor riesgo de muerte por lesiones de tránsito en barrios de menor nivel socioeconómico ${ }^{4}$.

Los estudios ecológicos que investigaron el rol de factores contextuales sobre las muertes por lesiones de tránsito hallaron que tanto factores del ambiente físico (áreas con alta sinuosidad de la red de caminos) como del ambiente social (áreas con menor densidad poblacional, menor nivel socioeconómico, mayor desempleo, baja disponibilidad de servicios de emergencia médica, mayor volumen de tráfico y mayor consumo de alcohol) estuvieron relacionados a mayores tasas de mortalidad por lesiones de tránsito ${ }^{5-11}$.

La mayoría de estos estudios fueron realizados en países desarrollados, por lo que el efecto de determinados factores causales contextuales podría diferir en países subdesarrollados con grandes disparidades regionales, como es el caso de Argentina. Además de estas disparidades, Argentina ha experimentado grandes fluctuaciones económicas durante las últimas décadas. Hacia finales de la década del noventa comenzó un período de recesión económica de cuatro años que culminó en 2002. Luego de esta crisis se inició un período de importante crecimiento económico que llevó a una disminución constante de los niveles de desempleo y un mayor nivel de consumo en la población, también reflejado en mayores niveles de motorización ${ }^{12}$. A este mayor nivel de exposición al tránsito en Argentina pudieron haberse sumado otros factores durante el periodo de crecimiento económico (como mayores niveles de consumo de alcohol, menor tiempo para dormir, trabajo excesivo) que pudieron acrecentar el riesgo de muerte por lesiones de tránsito ${ }^{13}$ en algunas áreas del país. Por lo tanto, sería interesante analizar si existieron cambios en los patrones de distribución geográfica de la mortalidad por lesiones de tránsito entre estos dos períodos.

A escala global, se registra un gradiente ascendente de mortalidad en ocupantes de vehículos de cuatro ruedas a medida que aumenta el ingreso per capita de los países, mientras que se registra un patrón inverso en el caso de los usuarios vulnerables de vías de tránsito (peatones, ciclistas y motociclistas) ${ }^{14}$. Sin embargo, se sabe muy poco acerca de los patrones de distribución espacial de las muertes por lesiones de tránsito para diferentes tipos de usuarios a nivel nacional. Los trabajos existentes son contados y enfocados en algunos usuarios de vías de tránsito ${ }^{15,16}$. En Argentina, un país con grandes disparidades territoriales ${ }^{17}$ y configurado espacialmente con un centro geográfico de mayor desarrollo económico (región Pampeana) con respecto a zonas mixtas o periféricas intermedias (principalmente regiones de Cuyo y Patagonia) y periféricas rezagadas (mayor parte del norte del país), se espera que el patrón mundial de distribución de la mortalidad de diferentes usuarios de vías de tránsito se reproduzca al interior del país. Es decir, un mayor riesgo de mortalidad de ocupantes de vehículos de cuatro ruedas en el centro geográfico y un mayor riesgo de mortalidad de usuarios vulnerables de vías de tránsito de las zonas mixtas y periféricas de la Argentina. 
Salvo algunas excepciones ${ }^{9,18}$, los estudios que han analizado los factores contextuales de área que inciden en la ocurrencia de muertes por lesiones de tránsito no han incorporado la dimensión espacial. De acuerdo a la primera ley de la Geografía, la ley de Tobler, "todo está relacionado (con todo lo demás), pero las cosas más cercanas están más relacionadas que las distantes"19. La presencia de asociación espacial en las muertes de diferentes usuarios de vías de tránsito nos indicaría la presencia de conglomerados de alta mortalidad y procesos de difusión espacial durante períodos de crecimiento económico. La difusión espacial ocurre cuando un fenómeno se propaga de un lugar a otro $^{20}$. Factores como el nivel de desarrollo económico de los territorios o pautas culturales relacionadas al uso de determinados medios de transporte pueden manifestarse a escala regional, superando los límites de una ciudad, una red de ciudades o límites provinciales al interior de un país. Es así como se considera a la escala geográfica como el producto de la acción social y, por lo tanto, mutable en el tiempo ${ }^{21}$.

Los objetivos de este artículo son: (a) analizar la distribución espacial del riesgo de mortalidad en diferentes tipos de usuarios de vías de tránsito en Argentina durante los períodos 1999-2003 y 2008-2012; y (b) analizar el rol de diferentes tipos de factores de área (socio-demográficos y relacionados al tráfico de vehículos) sobre el riesgo de mortalidad de diferentes usuarios de vías de tránsito.

\section{MÉTODO}

Se trabajó con datos aportados por la Dirección de Estadísticas e Información de Salud del Ministerio deSalud dela Nación (DEIS-MSAL). Fueronanalizados los datos demortalidad porlesiones de tránsito, de acuerdo a los códigos de la Clasificación Internacional de Enfermedades, Décima Revisión (CIE-10). Se consideraron los siguientes usuarios de vías de tránsito (cuarto código entre corchetes): peatones (V01-06 [1] y V09 [2-3]); ciclistas (V10-V18 [3-5,9] y V19 [4-6,9]); ocupantes de motocicletas (V20-28 [3-5,9] y V29 [4-6,9]); ocupantes de automóviles (V40-48 [4-7,9] y V49 [4-6,9]); y usuarios no especificados (V87 [0-9] y V89 [2-3, 9]). Se seleccionaron estos usuarios de vías de tránsito ya que constituyen los casos de mortalidad por lesiones de tránsito más frecuentes en Argentina. Para cada víctima por lesiones de tránsito se tomó en cuenta el lugar de ocurrencia del hecho fatal. La población en riesgo estuvo compuesta por la población residente en cada departamento, con base en datos de los censos nacionales de población (2001 y 2010). Las unidades espaciales $(n=512)$ estuvieron conformadas por las mínimas subdivisiones territoriales a nivel nacional (denominadas "partidos" en la Provincia de Buenos Aires y "departamentos" en el resto de las provincias argentinas) con datos disponibles sobre mortalidad, durante los períodos 1999-2003 y 2008-2012. La Ciudad Autónoma de Buenos Aires fue representada como una unidad espacial, debido a que las bases de mortalidad del DEIS-MSAL no contienen información desagregada sobre el lugar de ocurrencia de las defunciones para los distritos escolares que la componen (actualmente denominados 'comunas' y con diferentes límites geográficos). La elección de ambos quinquenios, 1999-2003 y 2008-2012, se debió a que estuvieron centrados en los dos últimos censos nacionales de población de 2001 y 2010. Si bien la adopción de ambos quinquenios se justifica por la disponibilidad de datos sociodemográficos relevados en toda la población argentina durante los censos, ambos periodos representan contextos socioeconómicos diferentes, marcados por años de recesión económica antes y después de $2001^{22}$ y mejoras sustanciales en los niveles de empleo y consumo en los años cercanos a $2010^{12}$ en relación al primer periodo (1999-2003).

La razón de mortalidad, calculada entre los casos observados de cada usuario de vías de tránsito durante los períodos 1999-2003 y 2008-2012 y los casos esperados, fue utilizada como indicador de mortalidad. Los casos esperados de mortalidad fueron calculados considerando la población total residente multiplicada por cinco en cada unidad espacial, de acuerdo a datos de los censos nacionales de 2001 y $2010^{23}$, por las tasas de mortalidad de cada usuario de vías de tránsito en Argentina.

Se formuló una regresión ecológica asumiendo una distribución de Poisson inflada en $\operatorname{ceros}^{24}$ en las muertes de cada usuario de vías de tránsito, debido a la presencia de sobredispersión causada por un excesivo número de ceros. Se empleó el modelo propuesto 
por Besag-York-Mollie (BYM) para incorporar la dependencia espacial entre las áreas ${ }^{24}$. Es así como se incluye un parámetro que toma en cuenta los residuos espacialmente estructurados más un parámetro de residuos no estructurados. Los residuos espacialmente estructurados fueron calculados a través de una matriz de contigüidad espacial, en donde el criterio de vecindad fue determinado si un área compartía un límite con otra área. Se especificaron distribuciones a priori mínimamente informativas en el logaritmo de precisión del efecto no estructurado ( $\tau v) \sim \log$ Gamma $(1,0,0005)$, y el logaritmo de precisión del efecto estructurado (Tu) logGamma $(1,0,0005)^{24}$.

Se calcularon los riesgos relativos tomando en cuenta los dos efectos aleatorios. Luego de tomar en cuenta las variables independientes incluidas en el modelo, se calcularon los riesgos relativos residuales. Tanto los riesgos relativos como los riesgos relativos residuales fueron considerados significativamente menores o mayores que 1 si los intervalos de credibilidad del $95 \%$ no incluyeron el 1 . Por último, se calculó la probabilidad posterior de encontrar un riesgo relativo residual mayor a 1.

Fueron incluidos dos conjuntos de variables independientes:

- Variables sociodemográficas: se incluyeron la densidad poblacional (individuos por $\mathrm{km}^{2}$ ) y un índice de pobreza (nombre de la variable:'Pobreza'). Este índice fue calculado mediante la suma de los puntajes " $z$ " de las siguientes variables: porcentaje de personas en situación de hacinamiento (hogares con más de 3 personas por cuarto); porcentaje de jefes de hogar clasificados como 'obrero o empleado,' trabajador por cuenta propia'o 'trabajador familiar' en la variable de categoría ocupacional; porcentaje de personas residiendo en viviendas de baja calidad (individuos residiendo en viviendas clasificadas como CALMAT (calidad de los materiales) en las categorías IV y V durante el censo nacional de población de 2001, e INMAT en las categorías III y IV durante el censo nacional de población de 2010). En el caso de la variable referida a la calidad de las viviendas, ambas clasificaciones de los censos de 2001 y 2010 son análogas. Con base en los antecedentes mencionados anteriormente, se esperan mayores tasas de mortalidad en áreas de menor densidad poblacional $\left.\right|^{5,7}$ y mayores niveles de pobreza ${ }^{5,6,8,9,11}$. Con el objetivo de controlar por la estructura demográfica de las unidades espaciales, se realizó un análisis de componentes principales tomando las siguientes variables: porcentaje de población menor de 15 años de edad, porcentaje de población entre 15 y 34 años, porcentaje de población entre 35 y 59 años, porcentaje de población mayor de 59 años y un índice de masculinidad (porcentaje de varones sobre el porcentaje de mujeres). El empleo del análisis de componentes principales buscó evitar la presencia de colinealidad en las variables independientes a través de una reducción de datos. De dicho análisis se extrajeron los primeros dos componentes, ya que explicaron el $78 \%$ y $81 \%$ de la varianza total de las puntuaciones durante 2001 y 2010 , respectivamente (Tabla 1). El componente demográfico 1 durante 2001 estuvo caracterizado por áreas con baja proporción de población de menos de 35 años de edad, alta proporción de población mayor de 34 años de edad y un alto índice de masculinidad, mientras que durante 2010 reflejó de manera inversa estas características. El componente demográfico 2 estuvo caracterizado por áreas con baja proporción de población entre 15 y 59 años y un alto índice de masculinidad durante 2001. Durante 2010 este componente estuvo caracterizado por áreas con baja proporción de población joven, alta proporción de población de 35 a 59 años de edad y un índice alto de masculinidad (Tabla 1). El análisis de componentes principales fue realizado con el programa Stata versión 13.1 (StataCorp, College Station, Texas, Estados Unidos).

- Variables de tránsito de vehículos: la tasa de patentamiento de vehículos automotores (nombre de la variable: 'Patentamiento de autos') y la tasa de patentamiento de motocicletas ('Patentamiento de motos') (ambas por 1.000 habitantes) fueron calculadas para los años 2001 y 2010, siendo utilizadas como variables independientes durante los quinqueños 1999-2003 y 2008-2012, respectivamente. Ambas tasas fueron construidas con datos de la Dirección Nacional del Registro de la Propiedad Automotor (D.N.R.P.A.) (datos de patentamiento automotor y de motocicletas) y los censos nacionales de 
Tabla 1. Resultados de los análisis de componentes principales utilizando variables demográficas de los censos nacionales de población. Argentina, 2001 y 2010

\begin{tabular}{lccccc} 
& \multicolumn{2}{c}{ Censo 2001 } & & \multicolumn{2}{c}{ Censo 2010 } \\
\cline { 6 - 6 } \cline { 5 - 6 } & $\begin{array}{c}\text { Componente } \\
\text { demográfico 1 }\end{array}$ & $\begin{array}{c}\text { Componente } \\
\text { demográfico 2 }\end{array}$ & $\begin{array}{c}\text { Componente } \\
\text { demográfico 1 }\end{array}$ & $\begin{array}{c}\text { Componente } \\
\text { demográfico 2 }\end{array}$ \\
\hline Población $\leq 14$ años & $-0,54$ & 0,24 & & 0,52 & $-0,16$ \\
\hline Población 15-34 años & $-0,32$ & $-0,72$ & & 0,41 & 0,11 \\
\hline Población 35-59 años & 0,52 & $-0,18$ & $-0,49$ & 0,32 \\
\hline Población $\geq 60$ años & 0,55 & 0,20 & $-0,54$ & $-0,09$ \\
\hline Índice de masculinidad & $-0,20$ & 0,59 & 0,15 & 0,92 \\
\hline Valor propio & 2,97 & 0,96 & 3,03 & 0,99 \\
\hline Variabilidad explicada (\%) & 59 & 19 & 61 & 20 \\
\hline
\end{tabular}

población de 2001 y 2010. Se utilizaron estas variables debido a que no se encuentran disponibles los datos del parque de vehículos y motos a un nivel inferior al de las provincias argentinas. Se espera que tanto el patentamiento de automóviles como de motocicletas se relacionen positivamente a mayores tasas de mortalidad en usuarios de automóviles y motocicletas, respectivamente. Esta hipótesis se basa en estudios que solo analizaron tasas de mortalidad generales por lesiones de tránsito ${ }^{8,11}$.

Los análisis de regresión espacial se llevaron a cabo utilizando el paquete INLA en el programa $\mathrm{R}$ (versión R.3.2.5), mientras que el mapeo de los riesgos relativos, riesgos relativos residuales y probabilidades posteriores se realizó con el programa QGIS (versión 2.14.3).

\section{RESULTADOS}

Las Figuras 1-5 muestran la distribución geográfica de los riesgos relativos, riesgos relativos residuales y probabilidades posteriores de riesgos relativos residuales mayores a 1 . En el caso de la mortalidad de peatones, se registra un patrón similar de riesgo de mortalidad entre ambos períodos, aun considerando las variables independientes. Aunque se observó principalmente un área de alta mortalidad en la mitad oeste de la Argentina (Figura 1), el cálculo de las probabilidades posteriores mostró conglomerados más acotados geográficamente (Figuras 1C y 1F). Entre los períodos 1999-2003 y 2008-2012 se observó una contracción geográfica en las regiones de Cuyo y norte patagónico y este de Buenos Aires. Por otro lado, se observó una expansión geográfica en el noroeste argentino y Entre Ríos (Figuras 1C y 1F).

De manera similar a la mortalidad de peatones, la mortalidad de usuarios de bicicletas registró una contracción geográfica del área de alta mortalidad localizada en las regiones de Cuyo y norte patagónico entre 1999-2003 y 2008-2012, y una expansión geográfica en Chaco y Santa Fe entre ambos períodos (Figura 2).

En cuanto a la mortalidad de usuarios de motocicletas, si bien el área de alto riesgo relativo de mortalidad se contrajo entre 1999-2003 y 2008-2012, el cálculo de las probabilidades posteriores de riesgos relativos residuales mayores de 1 mostró una difusión espacial del riesgo de muerte hacia el centro norte de la Argentina durante el período 2008-2012, luego de controlar por las variables independientes (Figura 3).

En el caso de la mortalidad de usuarios de automóviles durante el período 1999-2003 se observó un riesgo relativo de mortalidad alto en el centro y sur de la Argentina, inclusive luego de incorporar las covariables (Figuras 4B y 4C). Durante el período 2008-2012 se observa el mismo patrón de distribución espacial, más la emergencia de otros conglomerados de alto riesgo relativo en el centro y norte de Argentina (provincias de Misiones, Jujuy y este de Entre Ríos) (Figuras 4Ey 4F). 
Período 1999-2003
A

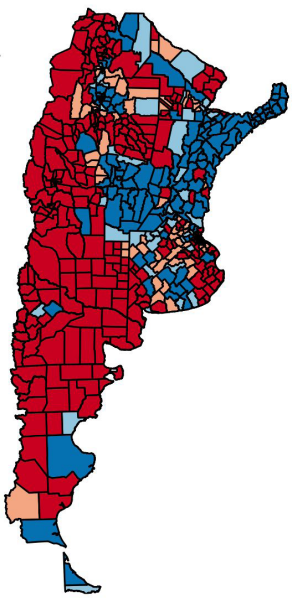

Riesgo relativo

$0,1-0,9$
$\square \quad 0,9-1,0$
$\square \quad 1,0-1,1$
$\square 1,1-16,5$

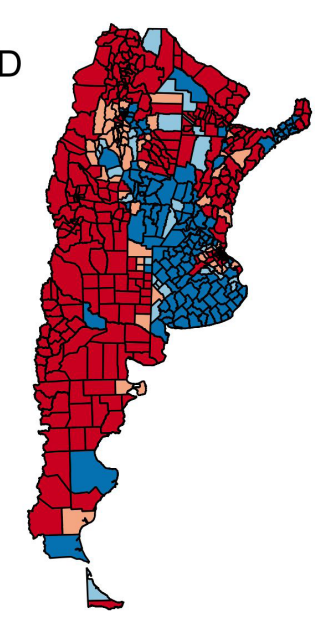

Riesgo relativo

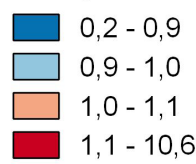

$\mathrm{B}$

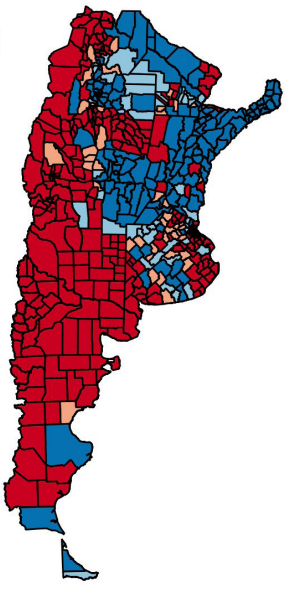

Riesgo relativo residual

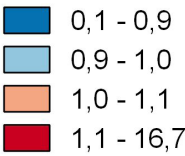

Período 2008-2012

E

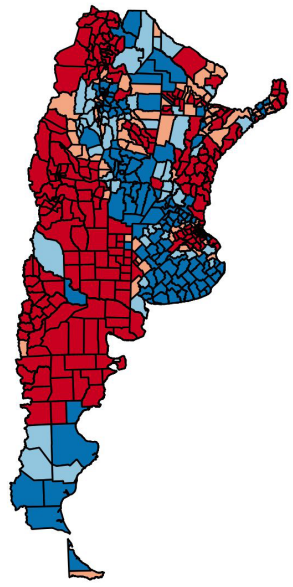

Riesgo relativo residual

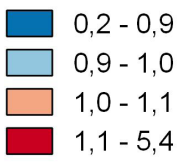

C

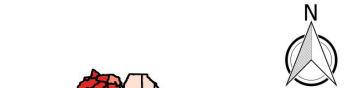

象

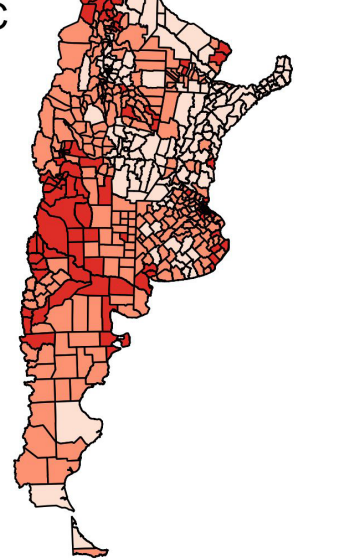

Probabilidad posterior

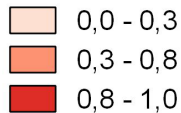

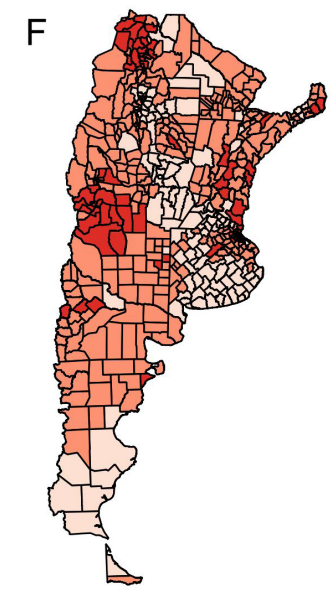

Probabilidad posterior

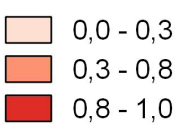

Figura 1. Distribución geográfica del riesgo relativo de mortalidad de peatones $(A, D)$, riesgo relativo residual $(B, E)$ (luego del ajuste por las variables independientes del modelo) y probabilidad posterior de riesgo mayor a 1 (C, F). Argentina, 1999-2003 y 2008-2012

Por último, la mortalidad de usuarios no especificados de vías de tránsito no mostró grandes variaciones espaciales entre los períodos 1999-2003 y 2008-2012 (Figura 5). En algunas provincias, como Entre Ríos, Córdoba y Neuquén, se observaron bajas probabilidades de riesgo relativo de muerte mayor a 1 durante 2008-2012 con respecto al período 1999-2003 (Figuras $5 C$ y $5 F$ ). 


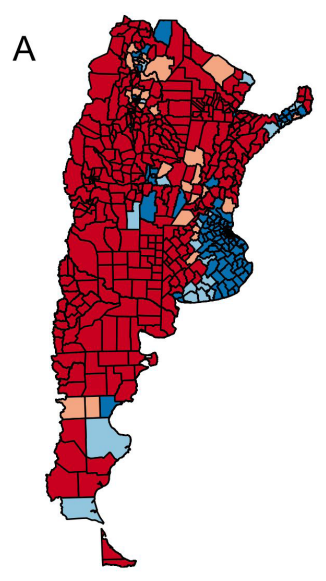

Riesgo relativo
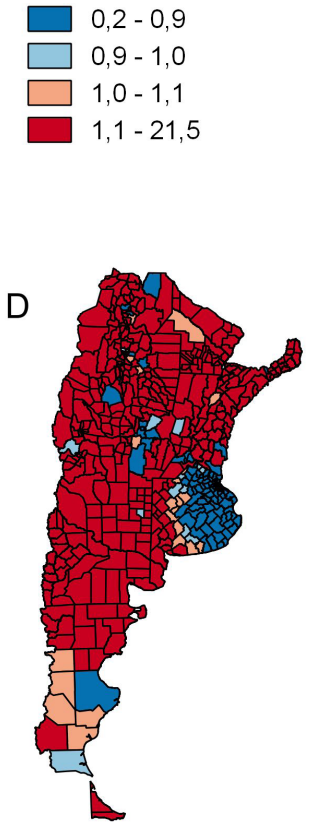

Riesgo relativo

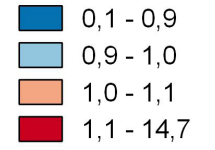

Período 1999-2003

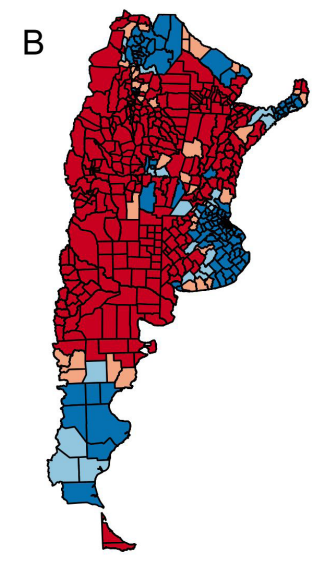

Riesgo relativo residual

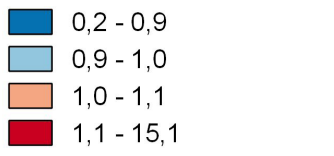

Período 2008-2012

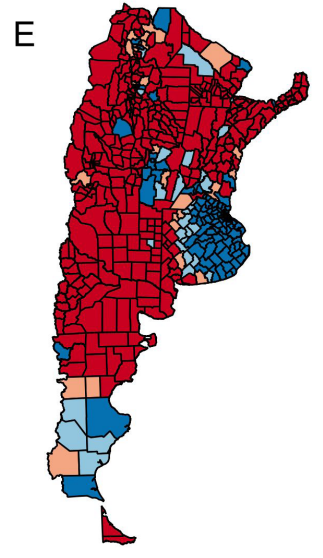

Riesgo relativo residual

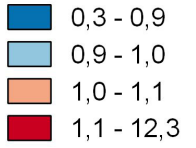

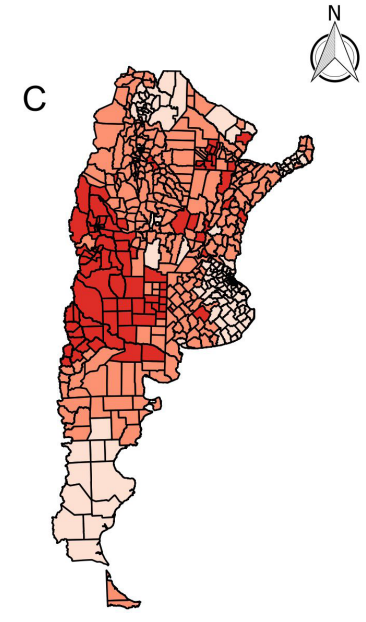

Probabilidad posterior

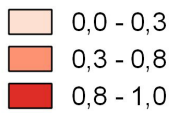

F

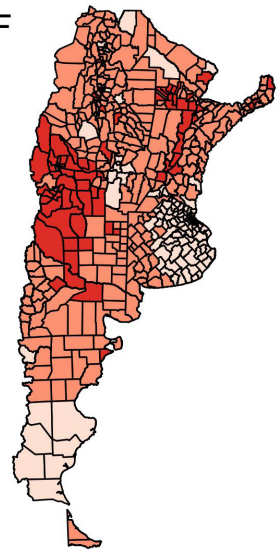

Probabilidad posterior

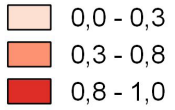

Figura 2. Distribución geográfica del riesgo relativo de mortalidad de usuarios de bicicleta $(A, D)$, riesgo relativo residual $(B, E)$ (luego del ajuste por las variables independientes del modelo) y probabilidad posterior de riesgo mayor a 1 (C, F). Argentina, 1999-2003 y 2008-2012

La Tabla 2 muestra los riesgos relativos de la mortalidad de cada usuario de vías de tránsito en relación a las variables independientes incluidas en los modelos ecológicos de regresión espacial. Cada riesgo relativo fue calculado controlando por el efecto de las demás variables independientes. Se exceptuó de la descripción de asociaciones significativas a los componentes demográficos ya que fueron incluidos como variables de control de la 

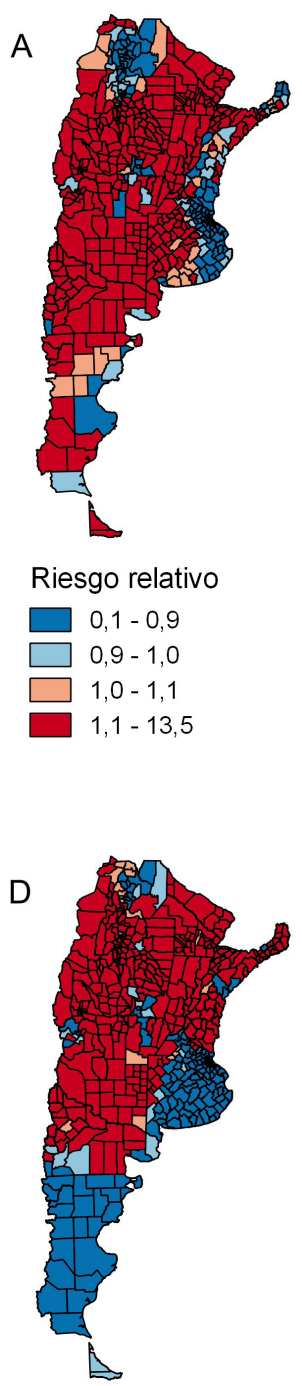

Riesgo relativo

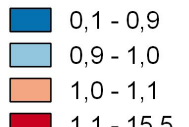

Período 1999-2003

B

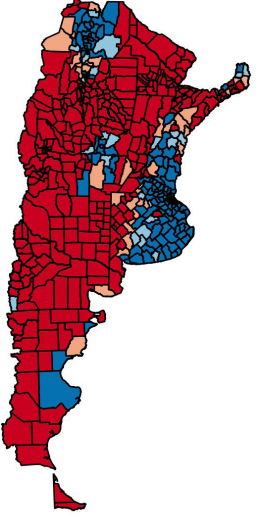

Riesgo relativo residual

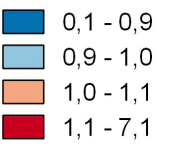

Período 2008-2012

$\mathrm{E}$

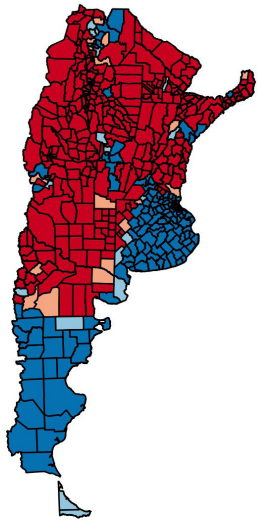

Riesgo relativo residual

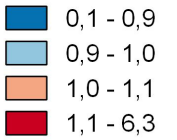

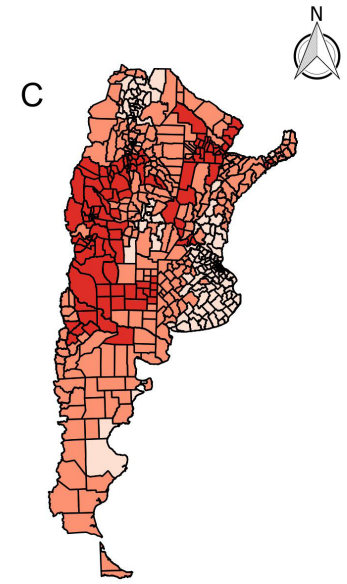

Probabilidad posterior

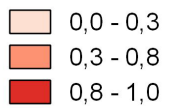

$0,8-1,0$

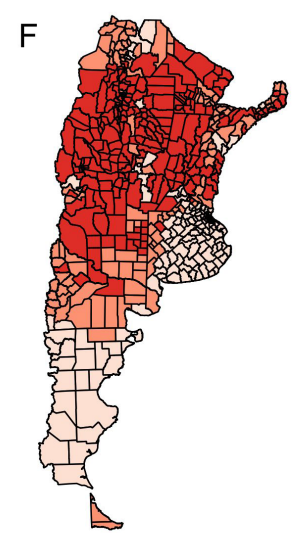

Probabilidad posterior

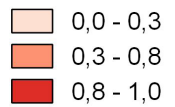

Figura 3. Distribución geográfica del riesgo relativo de mortalidad de usuarios de motocicleta $(A, D)$, riesgo relativo residual $(B, E)$ (luego del ajuste por las variables independientes del modelo) y probabilidad posterior de riesgo mayor a 1 (C, F). Argentina, 1999-2003 y 2008-2012

estructura demográfica. En el caso de los usuarios de bicicletas, la tasa de patentamiento de automóviles (solo durante 1999-2003: $\mathrm{RR}=1,06$; IC 95\%, 1,02-1,10) y el índice de pobreza (periodo 1999-2003: RR=1,17; IC 95\%, 1,02-1,33; periodo 2008-2012: $R R=1,19$; IC 95\%, $1,05-1,35$ ) se relacionaron positivamente con el riesgo de mortalidad (Tabla 2). Con respecto a los peatones, solo el índice de pobreza (periodo 2008-2012: $R R=1,21$; IC 95\%, 1,10-1,33) estuvo relacionado positivamente con un mayor riesgo de muerte. En el período 2008-2012 la densidad poblacional alta se asoció a un menor riesgo de mortalidad en ciclistas $(R R=0,82$; IC $95 \%, 0,71-0,94)$. Con respecto a los usuarios de motocicletas, la tasa de patentamiento de 
A

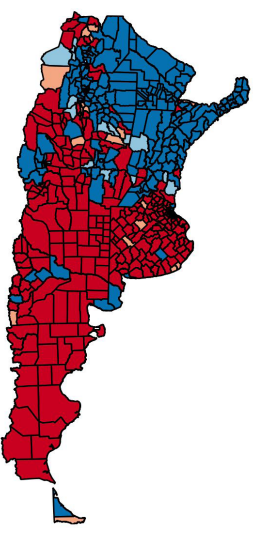

Riesgo relativo
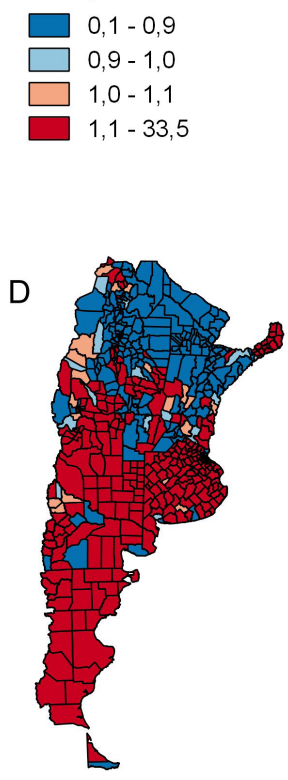

Riesgo relativo

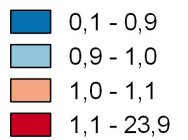

Período 1999-2003

B

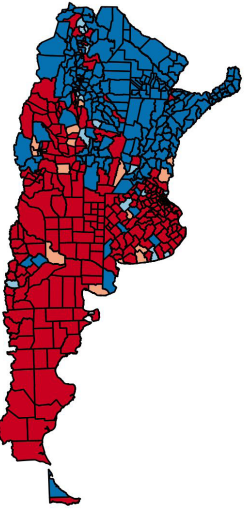

Riesgo relativo residual

$\square 0,1-0,9$

$0,9-1,0$

$1,0-1,1$

$1,1-22,9$

Período 2008-2012

E

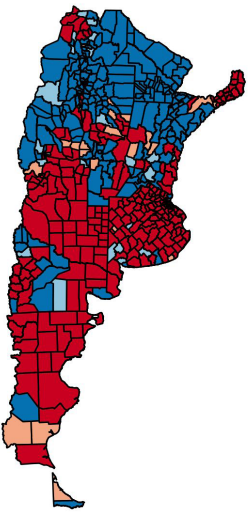

Riesgo relativo residual

$\square, 0,1-0,9$
$\square, 9-1,0$
$\square \quad 1,0-1,1$
$\square \quad 1,1-18,4$
C

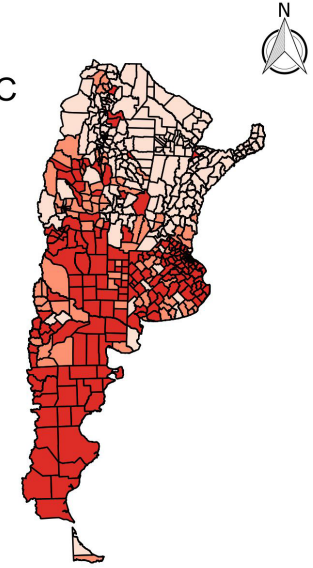

Probabilidad posterior
$0,0-0,3$
$0,3-0,8$
$0,8-1,0$

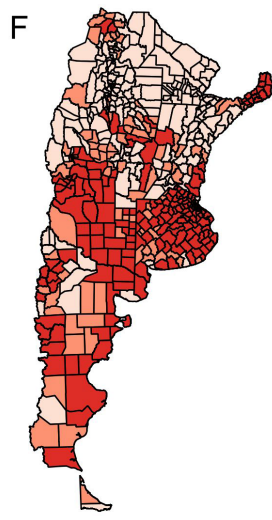

Probabilidad posterior

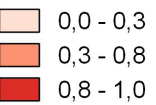

Figura 4. Distribución geográfica del riesgo relativo de mortalidad en usuarios de automóvil (A, D), riesgo relativo residual $(B, E)$ (luego del ajuste por las variables independientes del modelo) y probabilidad posterior de riesgo mayor a 1 (C, F). Argentina, 1999-2003 y 2008-2012

motos se asoció a un mayor riesgo de mortalidad solo durante 1999-2003 ( $R R=1,12$; IC 95\%, 1,04-1,20).

En el caso de los usuarios de automóviles, el riesgo de mortalidad fue mayor en áreas de menor densidad poblacional durante ambos períodos (periodo 1999-2003: RR=0,84; IC 95\%, 0,75-0,93; periodo 2008-2012: RR=0,78; IC 95\%, 0,70-0,86). Por último, en las áreas de menor densidad poblacional $(\mathrm{RR}=0,79 ; \mathrm{IC} 95 \%, 0,66-0,95)$ y con índices más bajos de pobreza ( $R R=0,87$; IC 95\%, 0,77-0,97) se observó un menor riesgo de mortalidad en usuarios no especificados de vías de tránsito durante 2008-2012 (Tabla 2). 


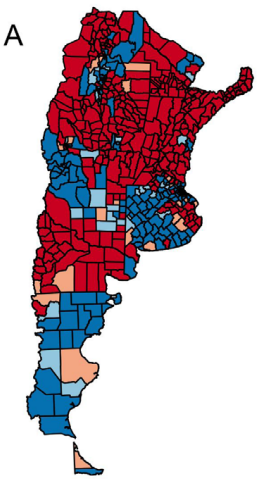

Riesgo relativo

$$
\begin{array}{r}
0,0-0,9 \\
\square \quad 0,9-1,0 \\
\square \quad 1,0-1,1
\end{array}
$$

$1,1-13,1$

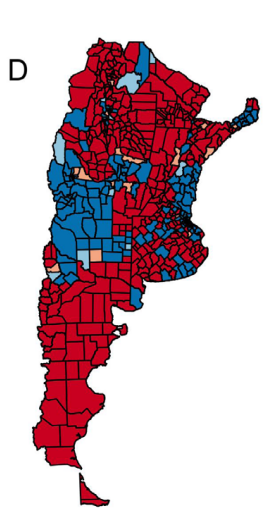

Riesgo relativo

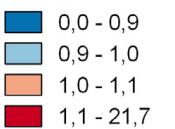

Período 1999-2003

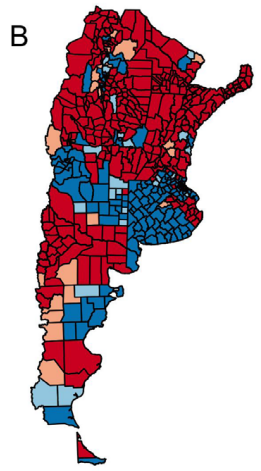

Riesgo relativo residual

$$
\begin{aligned}
& 0,1-0,9 \\
& 0,9-1,0 \\
& 1,0-1,1
\end{aligned}
$$

$1,1-11,5$

Período 2008-2012

E

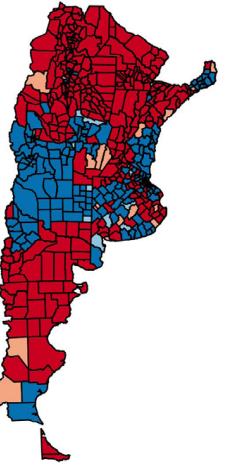

Riesgo relativo residual

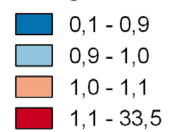

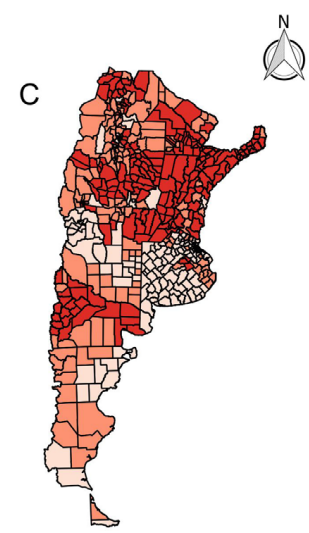

Probabilidad posterior

$\square \quad 0,0-0,3$

$0,8-1,0$

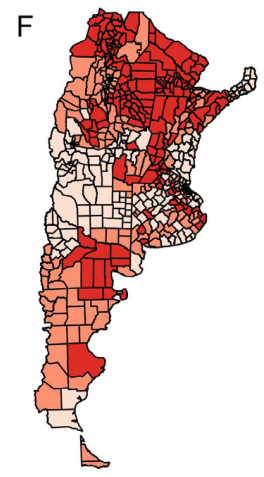

Probabilidad posterior

$$
\begin{aligned}
& 0,0-0,3 \\
& 0,3-0,8 \\
& 0,8-1,0
\end{aligned}
$$

\begin{tabular}{|c|c|c|c|}
\hline \multirow{2}{*}{$\begin{array}{l}\text { Usuarios de vías de } \\
\text { tránsito }\end{array}$} & \multirow{2}{*}{ Variables independientes } & \multicolumn{2}{|c|}{ Riesgo relativo (IC 95\%) } \\
\hline & & 1999-2003 & 2008-2012 \\
\hline \multirow{5}{*}{ Peatones } & Densidad poblacional & $1,03(0,93-1,15)$ & $0,98(0,89-1,07)$ \\
\hline & Índice de pobreza & $1,10(0,97-1,23)$ & $1,21(1,10-1,33)$ \\
\hline & Patentamiento de autos & $1,01(0,98-1,03)$ & $1,01(1,00-1,02)$ \\
\hline & Componente demográfico 1 & $1,07(0,93-1,23)$ & $0,87(0,77-0,99)$ \\
\hline & Componente demográfico 2 & $1,22(1,09-1,36)$ & $1,30(1,03-1,61)$ \\
\hline
\end{tabular}

Figura 5. Distribución geográfica del riesgo relativo de mortalidad en usuarios no especificados de vías de tránsito $(A, D)$, riesgo relativo residual $(B, E)$ (luego del ajuste por las variables independientes del modelo) y probabilidad posterior de riesgo mayor a 1 (C, F). Argentina, 1999-2003 y 2008-2012

Tabla 2. Riesgos relativos (e intervalos de credibilidad del 95\%) de las variables independientes. Argentina, 1999-2003 y 2008-2012 
Tabla 2. Continuación...

\begin{tabular}{|c|c|c|c|}
\hline \multirow{2}{*}{$\begin{array}{c}\text { Usuarios de vías de } \\
\text { tránsito }\end{array}$} & \multirow{2}{*}{ Variables independientes } & \multicolumn{2}{|c|}{ Riesgo relativo (IC 95\%) } \\
\hline & & 1999-2003 & 2008-2012 \\
\hline \multirow{5}{*}{ Ciclistas } & Densidad poblacional & $0,90(0,77-1,04)$ & $0,82(0,71-0,94)$ \\
\hline & Índice de pobreza & $1,17(1,02-1,33)$ & $1,19(1,05-1,35)$ \\
\hline & Patentamiento de autos & $1,06(1,02-1,10)$ & $1,01(1,00-1,02)$ \\
\hline & Componente demográfico 1 & $1,13(0,93-1,35)$ & $0,82(0,68-0,99)$ \\
\hline & Componente demográfico 2 & $1,22(1,04-1,41)$ & $1,10(0,72-1,61)$ \\
\hline \multirow{6}{*}{ Motociclistas } & Densidad poblacional & $0,93(0,82-1,06)$ & $1,02(0,91-1,15)$ \\
\hline & Índice de pobreza & $1,04(0,89-1,21)$ & $0,98(0,91-1,05)$ \\
\hline & Patentamiento de autos & $1,01(0,98-1,05)$ & $1,00(0,99-1,01)$ \\
\hline & Patentamiento de motos & $1,12(1,04-1,20)$ & $1,01(1,00-1,14)$ \\
\hline & Componente demográfico 1 & $1,11(0,91-1,33)$ & $1,03(0,92-1,16)$ \\
\hline & Componente demográfico 2 & $1,20(1,04-1,38)$ & $1,02(0,87-1,17)$ \\
\hline \multirow{5}{*}{ Usuarios de automóvil } & Densidad poblacional & $0,84(0,75-0,93)$ & $0,78(0,70-0,86)$ \\
\hline & Índice de pobreza & $1,03(0,94-1,12)$ & $1,05(0,97-1,14)$ \\
\hline & Patentamiento de autos & $1,01(0,99-1,03)$ & $1,00(0,99-1,00)$ \\
\hline & Componente demográfico 1 & $1,05(0,93-1,17)$ & $0,89(0,81-0,98)$ \\
\hline & Componente demográfico 2 & $1,35(1,22-1,48)$ & $1,39(1,22-1,46)$ \\
\hline \multirow{6}{*}{$\begin{array}{l}\text { Usuarios no } \\
\text { especificados de vías } \\
\text { de tránsito }\end{array}$} & Densidad poblacional & $0,93(0,81-1,06)$ & $0,79(0,66-0,95)$ \\
\hline & Índice de pobreza & $0,91(0,83-1,00)$ & $0,87(0,77-0,97)$ \\
\hline & Patentamiento de autos & $0,98(0,95-1,00)$ & $1,00(0,99-1,02)$ \\
\hline & Patentamiento de motos & $1,02(0,96-1,09)$ & $1,00(0,99-1,01)$ \\
\hline & Componente demográfico 1 & $1,00(0,88-1,14)$ & $1,06(0,88-1,25)$ \\
\hline & Componente demográfico 2 & $1,10(0,99-1,23)$ & $1,22(0,95-1,54)$ \\
\hline
\end{tabular}

\section{DISCUSIÓN}

Los resultados de este artículo indican que el riesgo de mortalidad de la mayoría de los usuarios de vías de tránsito se concentró espacialmente durante dos quinqueños en Argentina. Sin embargo, los conglomerados de alta mortalidad para cada modo de transporte se localizaron en diferentes partes de Argentina. Este hallazgo plantea la necesidad de llevar adelante políticas públicas diferenciales de prevención y control específicas en los conglomerados de alta mortalidad.

A pesar de estas diferencias en la distribución geográfica de la mortalidad, este estudio muestra cierta estabilidad espacial en la localización de áreas de alto riesgo de mortalidad durante dos períodos que abarcaron 15 años entre el comienzo del primero y el final del segundo (1998-2012). La difusión o contracción espacial del riesgo de mortalidad se dio en varios casos a partir de zonas de alta mortalidad persistentes en ambos períodos. Estos fenómenos fueron visibles en la mayoría de los conglomerados de mortalidad de los usuarios de vías de tránsito analizados en este estudio.

En combinación a esta persistencia de áreas de alta mortalidad durante ambos periodos, en este estudio se observó una distribución geográfica diferencial de la mortalidad de acuerdo 
al tipo de usuario de vías de tránsito. Esta distribución diferencial se podría relacionar con las disparidades territoriales persistentes en el desarrollo económico de la Argentina. Este patrón geográfico de desarrollo económico de centro, áreas mixtas, periferias intermedias y periferias rezagadas ${ }^{17}$ produciría un patrón geográfico de alta mortalidad de usuarios de automóviles principalmente en el centro y áreas mixtas, y alta mortalidad de usuarios vulnerables de vías de tránsito mayormente en periferias intermedias y rezagadas. Esto sería congruente con patrones diferenciales de distribución geográfica de la mortalidad hallados a nivel global ${ }^{14} \mathrm{y}$ a nivel intra-nacional en el caso de la mortalidad de peatones ${ }^{15}$.

Además, la estabilidad espacial en algunas áreas de alta mortalidad durante los dos períodos de estudio indicaría la presencia de procesos regionales, en el sentido de su extensión geográfica más allá de un departamento determinado, de carácter permanente. Estos procesos se combinarían a fenómenos cíclicos (crisis y crecimiento económico) que provocarían contracción y difusión espacial en los conglomerados de alta mortalidad. Además, los resultados de este estudio indicarían que estas variaciones geográficas son diferenciales entre usuarios motorizados y no motorizados. En general, mientras los peatones y usuarios de bicicletas mostraron una contracción espacial de áreas de alta mortalidad entre 1999-2003 y 2008-2012 (especialmente en el centro-oeste de la Argentina), los usuarios de motocicletas y automóviles mostraron mayormente una difusión geográfica de la mortalidad entre ambos períodos (a excepción de la Patagonia, en el caso de los automovilistas).

Whitelegg ${ }^{25}$ muestra que las medidas más tradicionales para disminuir los accidentes de tránsito se basan en el vehículo, el humano o el ambiente, pero éste último considerado solo en el contexto geográfico más próximo al siniestro vial. Este autor también muestra que las políticas de seguridad vial abarcan como máximo la escala de la ciudad, por lo que existiría un vacío referido a políticas explícitas a nivel regional, entendido como una red de ciudades y su matriz rural circundante.

Aunque no fue objetivo de esta investigación evaluar el efecto de las políticas de seguridad vial, la estabilidad general de los patrones de distribución geográfica encontrados en la mortalidad de los diferentes usuarios de vías de tránsito, entre 1999-2003 y 2008-2012, se produjo en un contexto de creación de la Agencia Nacional de Seguridad Vial en 2008. La creación de esta agencia implicó un aumento en los controles sobre el tránsito (instalación de radares para el control de la velocidad y pruebas de alcoholemia, especialmente en rutas nacionales), mayor capacitación a técnicos y funcionarios y un aumento de las campañas de concientización vial ${ }^{26}$. Aunque este aumento de las políticas de prevención y control pudo haber sido efectivo a nivel local, hay indicios de un mayor peso de los factores ligados al crecimiento económico en la difusión espacial de la mortalidad de usuarios motorizados (motociclistas y automovilistas).

Solo se observó un mayor riesgo de mortalidad en áreas de menor nivel socioeconómico en peatones y usuarios de bicicletas. Estos resultados se asemejan a los encontrados en los países desarrollados, donde registraron un mayor riesgo de mortalidad en las áreas de menor nivel socioeconómico $5,6,8,9,11$. En el caso de los países latinoamericanos, existen pocos antecedentes con resultados mixtos. Mientras en la ciudad de Recibe (Brasil) se encontró una tasa mayor de mortalidad por lesiones de tránsito en los barrios con mejores condiciones de vida $^{27}$, en Argentina se encontró el patrón inverso ${ }^{28}$.

El aumento importante en el patentamiento de automóviles y motos, debido al crecimiento económico experimentado durante el período 2003-2010, no solo estuvo relacionado a un aumento en la mortalidad en los mismos usuarios de esos vehículos, como fue el caso de los motociclistas, sino también podría haber impactado en otros usuarios vulnerables de vías de tránsito como los usuarios de bicicletas.

La bicicleta es un medio de transporte comúnmente utilizado para ir al trabajo, empleado mayormente por hombres de estratos socioeconómicos bajos y con poco uso de equipamientos de seguridad ${ }^{29}$. A esta situación de vulnerabilidad se agrega el recorrido obligatorio de los ciclistas por vías de tránsito dominadas por vehículos de motor: después de las causas inespecíficas, los ciclistas mueren más frecuentemente por colisiones con vehículos de cuatro ruedas $^{30}$. 
El riesgo de mortalidad en automovilistas y ciclistas se incrementó en áreas de baja densidad poblacional. Ambos resultados coinciden con lo estudiado en países desarrollados, registrando una relación negativa entre la mortalidad por lesiones de tránsito y la densidad poblacional $\left.\right|^{5,731,32}$. Otros estudios han analizado los factores que contribuyen a mayores niveles de mortalidad en áreas de baja densidad poblacional, considerando de manera agregada a todas las víctimas fatales por lesiones de tránsito o sólo los ocupantes de vehículos de motor. Entre los factores encontrados se puede mencionar una menor disponibilidad de servicios médicos de emergencia ${ }^{31}$, mayor grado de severidad de los accidentes fatales en áreas rurales ${ }^{33,34}$, o factores previos al accidente, como una estructura de la población más envejecida ${ }^{33,35}$

Con respecto al déficit de registro que generan las muertes de usuarios no especificados, resultan necesarios más planes de capacitación de recursos humanos en el sector de salud a nivel provincial, especialmente en las provincias de Chaco, Formosa, Jujuy, Santa Fe y Santiago del Estero.

Este estudio presenta algunas limitaciones. En primer lugar, se consideró el lugar de ocurrencia del evento independiente del lugar de residencia de la víctima. Por ejemplo, en departamentos de baja densidad poblacional pero con importantes flujos de transporte (departamentos en o con conexión a zonas turísticas) se pudo registrar una mayor frecuencia de muertes de individuos residentes en otros departamentos. En segundo lugar, la imposibilidad de incluir datos de mortalidad de las comunas que integran la Ciudad Autónoma de Buenos Aires no permitió analizar patrones de distribución espacial al interior de la ciudad para los diferentes tipos de usuarios de vías de tránsito.

En síntesis, se pueden sacar las siguientes conclusiones: en primer lugar, el riesgo de mortalidad de los usuarios de vías de tránsito analizados en este estudio se concentró en el espacio geográfico, con riesgos de mortalidad de un área determinada siendo similares a los riesgos de áreas vecinas. Segundo, en general se observó una estabilidad espacial en las áreas de alta mortalidad registradas en ambos períodos. Tercero, se observaron patrones de distribución geográfica diferenciales de la mortalidad de acuerdo a los usuarios de vías de tránsito analizados en este estudio que generalmente guardaron relación con las disparidades territoriales del desarrollo económico argentino. Por último, las variables ambientales utilizadas se asociaron diferencialmente con el riesgo de mortalidad de los usuarios de vías de tránsito y, a nivel de departamentos/partidos, el estatus socioeconómico bajo de área parece estar relacionado a un mayor riesgo de mortalidad en usuarios vulnerables no motorizados de vías de tránsito.

\section{REFERENCIAS}

1. Ubeda C, Espitia-Hardeman V, Bhalla K, Borse NN, Abraham JP, Dellinger A, et al. National burden of road traffic injuries in Argentina. Int J Inj Contr Saf Promot. 2012;19(1):9-18. http://dx.doi.org/10.1080/17457 300.2011.581377. PMid:21660797.

2. Haddon W Jr. Advances in the Epidemiology of Injuries as a Basis for Public Policy. Public Health Rep. 1980;95(5):411-21. PMid:7422807.

3. Laurell AC. Sobre la concepción biológica y social del proceso salud enfermedad. In: Rodriguez MI. Lo biológico y lo social - su articulación en la formación de personal de salud. Washington D.C.: Organización Panamericana de la Salud; 1994.

4. Cubbin C, LeClere FB, Smith GS. Socioeconomic status and the occurrence of fatal and nonfatal injury in the United States. Am J Public Health. 2000;90(1):70-7. http://dx.doi.org/10.2105/AJPH.90.1.70. PMid:10630140.

5. Baker SP, Whitfield RA, O'Neill B. Geographic variations in mortality from motor vehicle crashes. N Engl J Med. 1987;316(22):1384-7. http://dx.doi.org/10.1056/NEJM198705283162206. PMid:3574414.

6. Van Beeck EF, Mackenbach JP, Looman CWN, Kunst AE. Determinants of Traffic Accident Mortality in the Netherlands: A Geographical Analysis. Int J Epidemiol. 1991;20(3):698-706. http://dx.doi.org/10.1093/ ije/20.3.698. PMid:1955254.

7. Joly M-F, Thouez J-P, Bourbeau R, Bussière Y, Rannou A. Geographical variation in traffic-related mortality and morbidity among pedestrians in Quebec, 1983-1988. J Adv Transp. 1992;26(1):61-77. http://dx.doi. org/10.1002/atr.5670260106. 
8. Haynes R, Jones A, Harvey I, Jewell T, Lea D. Geographical distribution of road traffic deaths in England and Wales: place of accident compared with place of residence. J Public Health. 2005;27(1):107-11. http:// dx.doi.org/10.1093/pubmed/fdh212. PMid:15749726.

9. Aguero-Valverde J, Jovanis PP. Spatial analysis of fatal and injury crashes in Pennsylvania. Accid Anal Prev. 2006;38(3):618-25. http://dx.doi.org/10.1016/j.aap.2005.12.006. PMid:16451795.

10. La Torre G, Van Beeck E, Quaranta G, Mannocci A, Ricciardi W. Determinants of within-country variation in traffic accident mortality in Italy: a geographical analysis. Int J Health Geogr. 2007;6(1):49. http://dx.doi. org/10.1186/1476-072X-6-49. PMid:17956610.

11. Jones AP, Haynes R, Kennedy V, Harvey IM, Jewell T, Lea D. Geographical variations in mortality and morbidity from road traffic accidents in England and Wales. Health Place. 2008;14(3):519-35. http://dx.doi. org/10.1016/j.healthplace.2007.10.001. PMid:18032087.

12. Manzoni C. La venta de motocicletas batirá récords este año. La Nación [Internet]. 2007 [citado el 15 de marzo de 2019]; Disponible en: https://www.lanacion.com.ar/economia/la-venta-de-motocicletas-batirarecords-este-ano-nid955011

13. Tapia Granados JA. Recessions and Mortality in Spain, 1980-1997. Eur J Popul Rev Eur Démographie. 2005;21(4):393-422. http://dx.doi.org/10.1007/s10680-005-4767-9.

14. Naci $\mathrm{H}, \mathrm{Chisholm} \mathrm{D,} \mathrm{BakerTD.} \mathrm{Distribution} \mathrm{of} \mathrm{road} \mathrm{traffic} \mathrm{deaths} \mathrm{by} \mathrm{road} \mathrm{user} \mathrm{group:} \mathrm{a} \mathrm{global} \mathrm{comparison.}$ Inj Prev. 2009;15(1):55-9. http://dx.doi.org/10.1136/ip.2008.018721. PMid:19190278.

15. Paulozzi LJ. Is it safe to walk in the Sunbelt? Geographic variation among pedestrian fatalities in the United States, 1999-2003. J Safety Res. 2006;37(5):453-9. http://dx.doi.org/10.1016/j.jsr.2006.06.003. PMid:17112541.

16. Morais OL No, Montenegro MM, Monteiro RA, Siqueira JB Jr, Silva MM, Lima CM, et al. Mortalidade por acidentes de transporte terrestre no Brasil na última década: tendência e aglomerados de risco. Cien Saude Colet. 2012;17(9):2223-36. http://dx.doi.org/10.1590/S1413-81232012000900002. PMid:22996872.

17. Cao H, Vaca J. Desarrollo regional en la Argentina: la centenaria vigencia de un patrón de asimetría territorial. EURE Santiago. 2006;32(95):95-111. http://dx.doi.org/10.4067/S0250-71612006000100006.

18. Erdogan S. Explorative spatial analysis of traffic accident statistics and road mortality among the provinces of Turkey. J Safety Res. 2009;40(5):341-51. http://dx.doi.org/10.1016/j.jsr.2009.07.006. PMid:19932314.

19. Tobler WR. A computer movie simulating urban growth in the Detroit region. Econ Geogr. 1970;46:234-40. http://dx.doi.org/10.2307/143141.

20. Brown LA. Diffusion. In: Kitchin R, Thrift N, editors. International encyclopedia of human geography. Coventry: Elsevier; 2009. p. 170-84. http://dx.doi.org/10.1016/B978-008044910-4.00264-9.

21. Smith N. Remaking scale: competition and cooperation in prenational and postnational Europe. In: Eskelinen H, Snickars F. Competitive European Peripheries. Springer; 1995. p. 59-74. http://dx.doi. org/10.1007/978-3-642-79955-6_4.

22. CEPAL. Argentina: national economic profile [Internet]. 2017 [citado 2017 octubre 5]. Disponible en: http://interwp.cepal.org/cepalstat/Perfil_Nacional_Economico.html?pais=ARG\&idioma=english

23. Instituto Nacional de Estadística y Censos. WebINDEC: Población/Censos [Internet]. 2018 [citado 2018 noviembre 11]. Disponible en:https://www.indec.gob.ar/nivel3_default.asp?id_tema_1=2\&id_tema_2=41

24. Blangiardo M, Cameletti M. Spatial and spatio-temporal Bayesian models with R-INLA. Hoboken: John Wiley \& Sons; 2015. http://dx.doi.org/10.1002/9781118950203.

25. Whitelegg J. A geography of road traffic accidents. Trans Inst Br Geogr. 1987;12(2):161-76. http://dx.doi. org/10.2307/622525.

26. Argentina. Ministerio de Transporte. Agencia Nacional de Seguridad Vial [Internet]. 2017 [citado 2019 marzo 11]. Disponible en: https://www.argentina.gob.ar/seguridadvial

27. Lima MLC, Ximenes R. Violência e morte: diferenciais da mortalidade por causas externas no espaço urbano do Recife, 1991. Cad Saude Publica. 1998;14(4):829-40. http://dx.doi.org/10.1590/S0102311X1998000400025. PMid:9878916.

28. Macías GR. A complexidade da situação epidemiológica dos acidentes do transito: Uma análise da mortalidade por acidentes de transito, acontecida no Município de Lanús, Província de Buenos Aires, e na Argentina, entre os anos 1998 e 2004 [Tesis doctoral]. Salvador, Bahia: Universidade Federal da Bahia; 2009.

29. Bacchieri G, Gigante DP, Assunção MC. Determinantes e padrões de utilização da bicicleta e acidentes de trânsito sofridos por ciclistas trabalhadores da cidade de Pelotas, Rio Grande do Sul, Brasil. Cad Saude Publica. 2005;21(5):1499-508. http://dx.doi.org/10.1590/S0102-311X2005000500023. PMid:16158156. 
30. Galvão PVM, Pestana LP, Pestana VM, Spíndola MOP, Campello RIC, Souza EHA. Mortalidade devido a acidentes de bicicletas em Pernambuco, Brasil. Cien Saude Colet. 2013;18(5):1255-62. http://dx.doi. org/10.1590/S1413-81232013000500010. PMid:23670453.

31. Clark DE. Effect of population density on mortality after motor vehicle collisions. Accid Anal Prev. 2003;35(6):965-71. http://dx.doi.org/10.1016/S0001-4575(02)00104-5. PMid:12971931.

32. Lassarre $S$, Thomas I. Exploring road mortality ratios in Europe: national versus regional realities. J R Stat Soc Ser A Stat Soc. 2005;168(1):127-44. http://dx.doi.org/10.1111/j.1467-985X.2004.00340.x.

33. Maio RF, Green PE, Becker MP, Burney RE, Compton C. Rural motor vehicle crash mortality: the role of crash severity and medical resources. Accid Anal Prev. 1992;24(6):631-42. http://dx.doi.org/10.1016/00014575(92)90015-B. PMid:1388581.

34. Chen B, Maio RF, Green PE, Burney RE. Geographic variation in preventable deaths from motor vehicle crashes. J Trauma Acute Care Surg. 1995;38(2):228-32. http://dx.doi.org/10.1097/00005373-19950200000014. PMid:7869441.

35. Gedeborg R, Thiblin I, Byberg L, Melhus H, Lindbäck J, Michaelsson K. Population density and mortality among individuals in motor vehicle crashes. Inj Prev. 2010;16(5):302-8. http://dx.doi.org/10.1136/ ip.2009.024414. PMid:20595139. 